\title{
Das Spektrum der erregenden Absorption der Lenardphosphore.
}

\author{
Inaugural-Dissertation \\ zur Erlangung der Doktorwürde \\ der Hohen \\ Naturwissenschaftlich-Mathematischen Fakultät \\ der \\ Ruprecht-Karls-Universität \\ zu Heidelberg \\ vorgelegt am 1. Februar 1932 \\ von \\ Richard Kühner \\ aus Mannheim.
}


Tag der mündlichen Prüfung:

1. Februar 1932.

Erschienen in Annalen der Physik

5. Folge Band 13, Seite 873, 1932.

Gedruckt mit Genehmigung der naturwissenschaftlichmathematischen Fakultät der Universität Heidelberg.

\author{
Dekan: \\ Prof. Dr. K. Freudenberg \\ Referent: \\ Prof. Dr. Ferd. Schmidt

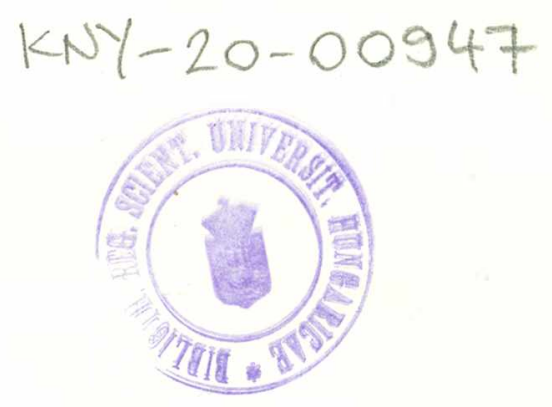




\section{Inhaltsübersicht.}

Die Aufgabe der vorliegenden Arbeit ist, den Charakter des Spektrums der erregenden Absorption der Lenardphosphore zu ermitteln und den Zusammenhang zwischen den Absorptionsfrequenzen und den Emissionsbanden näher zu ergründen. Die im Folgenden zusammengestellten Ergebnisse dieser Untersuchung sind an Beobachtungen des Spektrums erregender Absorption der $\mathrm{CaSCu}-, \mathrm{SrSCu}^{-}, \mathrm{CaSPb}-, \mathrm{SrSPb}-$ und $\mathrm{CaSMn}$-Phosphore erhalten.

I. Als eine Folge der kurzwelligen Tilgungswirkung auf die Zentren wird erkannt: 1. Alle untersuchten Phosphore zeigen mit Temperaturerhöhung eine schnellere Abnahme der Intensität der kurzwelligen Erregungsmaxima als der der langwelligen. 2. Mit wachsendem Metallgehalt nimmt die Ultravioletterregung ab. 3. Die maximale Erregung eines Bandenkomplexes liegt um so mehr im Ultravioletten je geringer der Metallgehalt des Phosphors ist.

11. Die den hohen Temperaturen angehörige Verteilung der erregenden Absorption der Phosphore wird als jene einer sehr schnell abklingenden Phosphoreszenz erkannt. Es werden dabei für sie bisher unbekannte Maxima gefunden.

III. Die Möglichkeit des Auftretens selektiver Maxima bei der Verteilung der sehr schnell abklingenden Phosphoreszenz ist genau so wie bei der Dauererregung gegeben.

IV. Für die Gesetzmäßigkeit, daß analoge Emmissionsbanden der Phosphore unabhängig von der Art des wirksamen Metalls in gleichem oder ungleichem Grundmaterial die annähernd gleiche Verteilung der erregenden Absorption zeigen, ergeben sich neue experimentelle Stützen.

V. Die von F. Schmi tt neu erkannten Gesetzmäßigkeiten ") erfahren eine experimentelle Bestätigung.

1) Vgl. F. Schmidt, Ann. d. Phys. 5. 12. S. 211. 1932. 


\section{Lebenslauf.}

Ich, Richard Kühner, wurde geboren zu Mannheim am 14. November 1908 als Sohn des Dekorateurs Georg Kühner. Ich bin evangelischer Konfession und badischer Staatsangehörigkeit. Vom 6. bis 9. Lebensjahr besuchte ich die Bürgerschule, vom 9. bis 18. Lebensjahr zunächst die Lessingschule und dann die Oberrealschule in Mannheim. Letztere verließ ich mit dem Zeugnis der Reife im Frühjahr 1927. Seit dem Sommersemester 1927 war ich an der Universität Heidelberg immatrikuliert und blieb hier bis zum Ende meines Studiums. Neben Physik studierte ich noch Chemie, Mathematik und Philosophie. Vorliegende Arbeit begann ich im Sommersemester 1930.

I ch besuchte die Vorlesungen und Praktika der Professoren und Dozenten:

Becker, Faust, Freudenberg, Glockner, Hieber, Lenard, Liebmann, E. Müller, M. Müller, Rosenthal, Salomon-Calvi, F. Schmidt, Strecker, Trautz, Wolf.

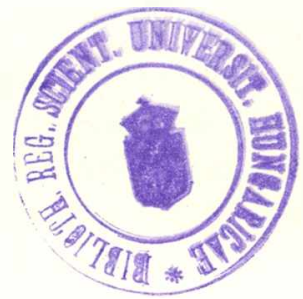

